\title{
Chaos detection and control in production systems
}

\author{
B. Scholz-Reiter, M. Freitag \\ Brandenburg Technical University of Cottbus \\ P.O. Box 101344, D-03013 Cottbus, Germany \\ Tel.: $+49355 / 694581$ \\ Fax: $+49355 / 694800$ \\ e-mail:bsr@iit.tu-cottbus.de
}

\begin{abstract}
The growing structural and dynamical complexity of today's production systems in combination with nonlinearities causes problems for production control. For analysing the complex production systems a production model is simulated with a production simulator and the dynamic system properties are examined with different methods of the linear and nonlinear time series analysis. The dependence of the stock fluctuations of different parameters and initial conditions are analysed. At the appearance of irregular stock fluctuations, suitable control methods should intrude into sensitive production sectors and lead the system back to a stable range.
\end{abstract}

Keywords

Production Control, Nonlinear Dynamics, Chaos Theory, Time Series Analysis

\section{INTRODUCTION}

The growing structural and dynamic complexity and nonlinearities in today's production systems leads to problems in production control. Conventional systems and strategies for production control can not cope with these issues, particularly when organisational sequences of operations are strongly integrated into a network and the planning horizon is very narrow. Small perturbations in the production flow can overshoot themselves and cause irregular system behaviour. This can be seen, for example, in extremely fluctuating stocks, which increase processing times. As a result, the prediction and meaningful control of the order processing becomes complicated. Methods from the field of nonlinear dynamics, which have been tested for their compatibility to production systems, are used for the analysis and control of nonlinear (chaotic) systems. As a result, an analysis of complex 
production systems should be possible even in the case of an irregular behaviour in order to determine unstable and endangered production sections and parameters and to derive corresponding control methods.

\section{MODELLING PRODUCTION SYSTEMS}

For the development of suitable analysis tools and controlling methods it is initially advisable to describe the behaviour of production systems with the help of dynamical models. There are two different approaches for modelling and analysis:

One can start with an element, a work system, describe it analytically and connect it via the order flow to the other work systems until complex networks arise.

Or, one can model and simulate complex production systems with standard production simulators and analyse the dynamics of the systems on the basis of the results of the simulation. This approach will be explained in the following.

The simulator FACTOR/AIM was selected as a basis for the work. The copying of real processes simulation models are built up on the basis of customer orders, working plans and production layouts from firms with workshop production. A simple production model with typical structures was built for the tests and extensive simulations were executed. Thereby, the inventory evolution in the buffers of the work systems is registered in the form of queue lengths. The temporal development of the stocks at the work system is determined by the input and the output in the viewed time period. The output depends on the processing time of the orders as well as on the capacity of the work system. The input arises from the output of the foregoing work systems or from newly released orders.

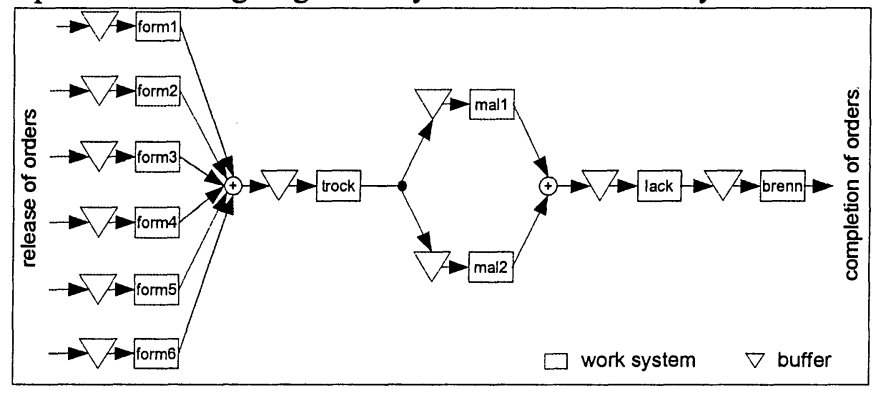

Figure 1 Production system „Ceramic manufacture“.

\section{ANALYSIS OF THE DYNAMIC SYSTEM PROPERTIES}

The dynamic properties of a system are generally determined from its dynamic equations. However, these are rarely available for real or realistically modelled systems. Therefore in many cases one must fall back on the measured data from the system. Such measurements can be the queue lengths of the buffers, in which 
the dynamics of the system are reflected. Figure 2 shows the plot of a stock time series, that resulted from a simulation with 6 periodical order releases.

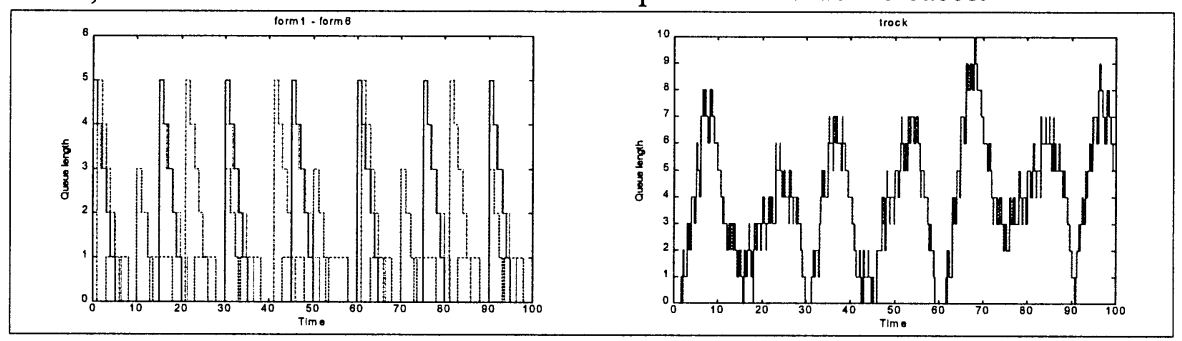

Figure 2 Queue length of work system ,trock“, developed from the simulation with 6 periodical order releases on the first work systems ,form1" to „form6“". The event orientated stock time series are sampled to equidistant times because many methods of the time series analysis suppose equidistant time series. From the sampled time series, the dynamic properties of the system such as periodicities, correlations and stochastic quotas can be determined by linear and nonlinear data analysis. Thus, control methods can be deduced at occurring irregularities.

\section{LINEAR TIME SERIES ANALYSIS}

Two „classical“ methods are initially used for the analysis of stock time series, the power spectrum and the autocorrelation function. As a first step, the Fourier analysis is used to distinguish between regular and irregular behaviour. Periodic oscillations are indicated by one or more sharp peaks, the fundamental frequency and its subharmonics. Quasiperiodic oscillations show two incommensurate frequencies. All further peaks consist of linear combinations from these fundamental frequencies. Aperiodic motions lead to broadband power spectra with many, no longer interpreting peaks. Power spectra of chaotic movements often have a high power density at low frequencies and can show a so-called $1 / \mathrm{f}$ behaviour.

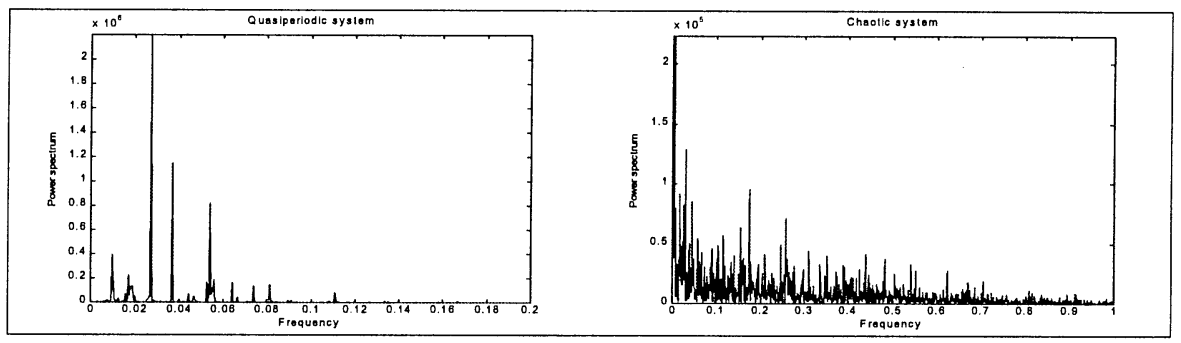

Figure 3 Power spectrum of a quasiperiodic and a chaotic system.

The autocorrelation function includes the temporal correlation of a time series with itself. That is, this function reflects the "memory“ of the system. In periodic systems the autocorrelation function contains only a proposition about the periodicity. In aperiodic systems it contains the information about the speed of the 
loss of the phase relation. The autocorrelation function of a chaotic oscillation decreases relatively quickly on function values around zero. The quicker the function decreases, the smaller inner correlations occur in the time series and a chaotic behaviour can be interpreted.

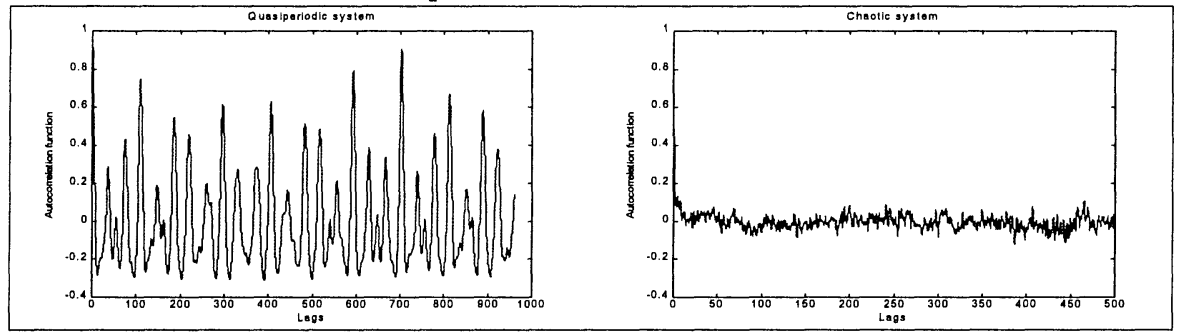

Figure 4 Autocorrelation function of a quasiperiodic and a chaotic system.

\section{NONLINEAR TIME SERIES ANALYSIS}

Nonlinear analysis methods are used for a better detection of irregularities and are tested for their compatibility in production systems. The analysis methods must be specified and developed for these conditions as production systems with their discrete order flow are not smooth systems

Firstly, linear interpolations of the discrete order flow were dealt with for the purpose of the tests. The interpolations correspond to the consideration of the indirect stocks, that is, they correspond to the forthcoming input of the foregoing work systems and the order's linear decreasing process time that is being processed.

\section{Attractor reconstruction}

The basis of the most nonlinear analysis methods is the representation of a time series in its phase space, the so-called attractor reconstruction. In the case of chaotic dynamics this leads to strange attractors, fractal constructs in the phase space, which can show very complicated but seemingly orderly geometrical forms at the same time. An examination of the geometrical structures of the attractor should give information about the systems behaviour.

Two different methods exist for the attractor reconstruction from onedimensional time series, the time delay method by Takens and the method of the singular value decomposition by Broomhead and King. The time delay method is very simple to use, but the choice of the delay time is difficult. The first zero crossing of the autocorrelation function or the first minimum of the automutual information is frequently used. The method of the singular value decomposition shows a few advantages over the time delay method, particularly with noisy data. 


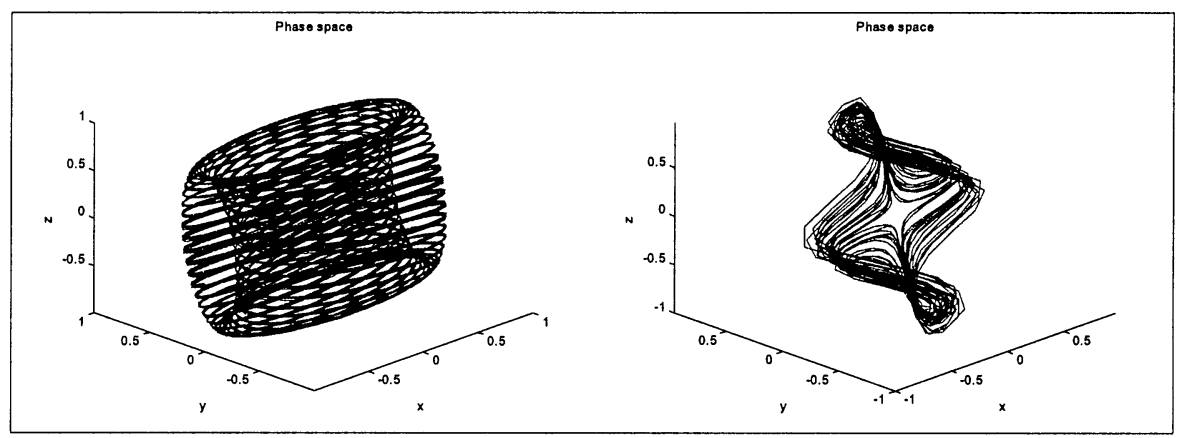

Figure 5 Reconstructed attractors, left from a quasiperiodic motion, right from a chaotic motion.

\section{Dimensions}

If one has successfully reconstructed an attractor then it is possible to estimate its dimension. The dimension characterises the geometry of an attractor, that is the dimension of its surface. This is one of its essential characteristics. A stationary process has the dimension zero, which is represented by a fix point in the phase space. A periodic motion shows a closed curve in the phase space with the geometrical dimension 1. The trajectories of a quasiperiodic motion cover the surface of a torus and have the dimension 2. However, chaotic attractors do not show any closed set of points. They present an open collection of points with a difficult, self-similar structure. The statistical distribution or density of these points in the phase space yields the fractal structure of a chaotic attractor, expressed by a noninteger dimension.

Periodical and quasiperiodical attractors therefore show integer dimensions, chaotic attractors are indicated by noninteger, fractal dimensions. Thus, the fractal dimension is seen as an essential criterion for the existence of deterministic chaos. However, this criterion is not sufficient, yet it can be interpreted as an indication to chaos.

\section{Lyapunov exponents and Kolmogorov entropy}

The most reliable indicator of chaos is at least one positive Lyapunov exponent. The Lyapunov exponent specifies the divergence rate of trajectories for every dimension of the phase space and thus the expansion of the effects of small disturbances and the sensitivity to small changes in initial conditions. Stable system states are characterised by negative Lyapunov exponents. A zero value indicates quasiperiodic behaviour. In the case of connected work systems, a set of local Lyapunov exponents can be specified indicating the chaotic points of the production system and the state evolution of the work systems (value of the exponent).

The sensitivity of work systems against parameter changes and the influence of parameter changes on the dynamics of several work systems is characterised by 
conditional Lyapunov exponents. Thus the points and parameters may be selected that are suitable for the control of the production system.

The Kolmogorov entropy is calculated by the integration of the positive local Lyapunov exponents over the phase space with consideration of the probability factor. It describes the system behaviour as a whole and is the most important measure for quantitative description of chaotic motion in the phase space. Its value equal or greater than zero characterises the actual stable or chaotic state of a production sector.

\section{Problems with nonlinear data analysis}

Several problems arose after conducting extensive tests with different nonlinear analysis algorithms:

- The algorithms require many data points. This supposes very long simulation times or in practice very long time periods for the operational data collection. Difficulties for practical use result from this.

- Measurement errors arise from the interpolation and the equidistant sampling of the stock time series, which become disturbing noticeably as additional noise. An exact determination of the dimensions, entropies and Lyapunov exponents was not reached.

- Many nonlinear analysis algorithms have been developed for smooth systems with continuous variables (observables). They are not compatible to systems with discrete variables (observables).

As a result, one presently works with the finely sampled, value discrete original time series. The two "classic“ methods, power spectrum and autocorrelation function, are used for the analysis.

\section{SENSITIVITY ANALYSES}

In nonlinear systems the dynamic stability properties are of primary interest. One tries to estimate this by sensitivity analyses. Unstable (chaotic) systems are indicated by a high sensitivity on changes of the initial conditions and the system parameters. The fluctuations of the stock levels at the work systems can be influenced strongly by tiny changes or perturbations of the order flow and then take a different course. Therefore the reactions of the autocorrelation functions and the power spectra of individual work systems are tested on parameter changes at definite sectors to analyse the stability of the system and to estimate the effect on the stock fluctuations of other work systems and on the complete system. With this, work systems should be found that have a strong influence on the dynamics in the production system or on definite work systems (e.g. bottlenecks). The following parameters and initial conditions can change within a production system:

- time and period of the release of orders,

- quantity of the orders, 
- processing duration of the orders,

- queue strategy,

- machine failure.

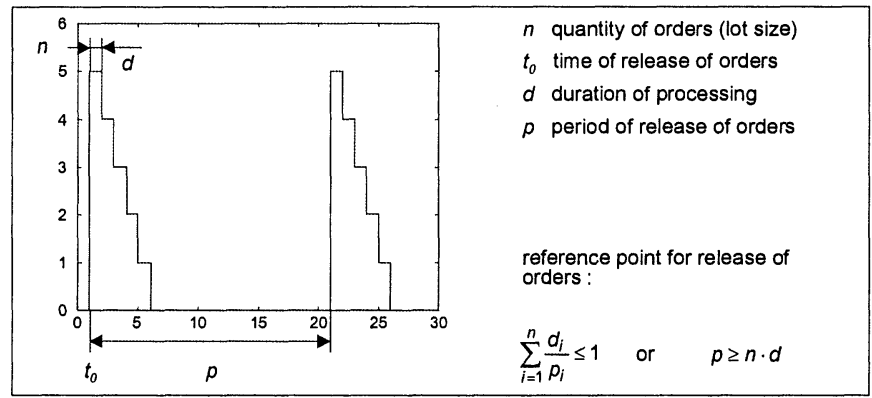

Figure 6 System parameters and initial conditions on the first work systems.

One finds work systems which have an intensive influence on the dynamics in the production system if the autocorrelation function and power spectrum of one or several work systems show the typical appearance for chaotic behaviour. The production system can only be controlled with small interventions in these sectors. Therefore they should also be very carefully supervised.

\section{CONCLUSION}

Today's production systems are often nonlinear and increasingly complex in regards to their dynamics. They can show irregularities and instabilities that reduce the predictability and controllability of the system and they can not cope with conventional methods.

An approach was demonstrated for the modelling of production processes and potentials to present methods of nonlinear dynamics for the analysis and control of stock fluctuations in production systems. The stability properties of these fluctuations and the consequences of changes of the production parameters can be estimated by sensitivity analysis.

As a result, an analysis of complex production systems is also possible with chaotic behaviour, so as to determine unstable and endangering production sections and parameter ranges and to derive corresponding control methods. A long-term aim is to develop methods which reduce buffer inventories, shorten throughput times and improve schedule effectiveness, even within the chaotic states of production systems. 


\section{REFERENCES}

Argyris, J., Faust, G. and Haase, M. (1994) Die Erforschung des Chaos. Vieweg Verlag, Braunschweig Wiesbaden.

Kantz, H. and Schreiber, T. (1997) Nonlinear Time Series Analysis. Cambridge University Press.

Kruel, T.-M. (1992) Zeitreihenanalyse nichtlinearer Systeme (Chaos und Rauschen). Dissertation an der Bayerischen Julius-Maximilians-Universität Würzburg.

Ott, E. (1993) Chaos in Dynamical Systems. Cambridge University Press.

Scholz-Reiter, B., Kleiner, M., Nathansen, K. and Proske, G. (1997) Chaos Control in Production Systems. Proceedings of the $15^{\text {th }}$ IMACS World Congress on Scientific Computation, Modelling and Applied Mathematics, Vol. 5, Wissenschaft \& Technik Verlag, Berlin, pp. 701-706.

Schuster, H.G. (1994) Deterministisches Chaos. VCH Weinheim.

Tönshoff, H. K. and Glöckner, M. (1994) Chaos-Phänomene in der Fertigung, wtProduktion und Management 84, pp. 255-258.

Wiendahl, H.-P. (1997) Fertigungsregelung: Logistische Beherrschung von Fertigungsabläufen auf Basis des Trichtermodells. Carl Hanser, München Wien.

\section{BIOGRAPHY}

Prof. Dr.-Ing. Bernd Scholz-Reiter studied Industrial Engineering at the Technical University of Berlin. He was then employed as a research fellow in the Department of Systems Analysis and Data Processing at the TU Berlin. He received his doctorate in 1990. From 1990 to 1991 he worked as an IBM World Trade Postdoctoral Fellow at the IBM T. J. Watson Research Center, Yorktown Heights, N.Y., USA, in the field of Manufacturing Research. After that he formed and supervised the teaching and research group Computer Aided Systems Analysis at the TU Berlin. In July 1994 he became Professor for Industrial Information Systems at the BTU Cottbus.

Dipl.-Ing. Michael Freitag studied Electrical Engineering at the BTU Cottbus specialising in Automation and Communication Technology. Since 1999 he has been working as a research fellow at the Chair of Industrial Information Systems at the BTU Cottbus.

\section{ACKNOWLEDGEMENT}

This research was founded by the Volkswagen Stiftung (I/73 515). 